\title{
Reliability and reproducibility of breast MRI as a roadmap in treatment planning of breast cancer-a single centre experience
}

\author{
Abstract \\ Aim: To study the significance of MRI in the preoperative work up of patients with \\ newly diagnosed breast cancer and its potential to create a road map for surgical and \\ definitive treatment. To study and define those interpretation criteria of MRI that serve \\ to increase its specificity in pre-treatment breast cancer assessment with emphasis \\ on non mass enhancement (NME). To study its role in predicting the nodal status in \\ diagnosed cases of breast cancer.
}

Material and methods: MRI and pathological data of 156 patients spread over period from September 2011 to JUNE 2014 were studied retrospectively. Morphology and enhancement characteristics of breast lesions, both masses and NME and axillary lymph nodes were analysed on DCE MRI with subsequent histopathological correlation.

Results: Our study showed Sensitivity (Sn): 79\%, Specificity (Sp): $86 \%$, Positive Predictive value (PPV): $76 \%$, Negative predictive value (NPV): $88 \%$ for identification of extratumoral DCIS (Ductal carcinoma in situ) while Sn: 74\%, Sp: 76, PPV: 52\%, NPV: $89 \%$ for extensive intraductal component (EIC) on histopathology in patients having non mass enhancement (NME) on DCE-MR. When NME was correlated with extratumoral DCIS and EIC taken together, we obtained Sn: 89\%, Sp: 78\%, PPV: 50\%, NPV: $97 \%$. Multifocal/multicentric tumours with or without satellite nodules on MRI had Sn: $86 \%$, Sp: $89 \%$, PPV: $60 \%$, NPV: $97 \%$ for multiple lesions on histopathology. For detection of axillary nodal metastasis, fatty hilum effacement showed Sn: $65 \%$, Sp: $89 \%$, PPV: $86 \%$, NPV: $73 \%$ and cortical thickening with and without fatty hilum effacement showed Sn: $86 \%$, Sp: $69 \%$, PPV: $73 \%$, NPV: $84 \%$.

Conclusion: MRI plays an important role in assessment of disease extent in breast cancer patients desirous of breast conservation surgery and has a potential to act as a road map in treatment planning. Following certain diagnostic criteria it helps in identifying the in situ cancers with a reasonable high specificity.

Keywords: multifocal/multicentric, non mass enhancement, axillary nodes, strategies, breast cancer, MRI, protocol, radiological, mammography, ultrasound, DCE, contra lateral, chemotherapy, hormonal therapy, extra tumoral
Volume 2 Issue 4 - 2017

Richa Bansal

Max Healthcare, India

Correspondence: Richa Bansal, Max Healthcare, Press Enclave Road, Saket II 00 I7, New Delhi, India,

Email richa1974@hotmail.com

Received: September 28, 2016 | Published: March 09, 2017
Abbreviations: MRI, magnetic resonance imaging; NME, non mass enhancement; Sn, sensitivity; Sp, specificity; PPV, positive predictive value; NPV, negative predictive value; SER, signal enhancement ratio; EIC, extensive intraductal component; BCT, breast-conservation therapy, DCIS, ductal carcinoma in situ

\section{Introduction}

Breast cancer is the second most common cause of cancer deaths among women, exceeded only by lung cancer. However the number of deaths reported has been decreasing mainly contributed by earlier detection through screening mammography and improved treatment strategies. ${ }^{1}$ Accurate staging of breast cancer is important in treatment planning and radiological findings are being incorporated into clinical staging as a routine protocol..$^{2-5}$ Screening mammography is used for early detection of breast cancer and Ultrasound is mainly used as a diagnostic modality and for guided biopsies. Contrast enhanced MRI along with Diffusion weighted imaging has come up to play a pivotal role in breast cancer staging in conjunction with mammography and ultrasound and can be used as a roadmap in treatment planning. ${ }^{6,7}$
DCE-MRI helps in detection of multicentric and multifocal disease in the same breast as well as in identification of synchronous lesions in contralateral breast. ${ }^{8}$ Involvement of the chest wall or pectoral muscle and the skin is better demonstrated by MRI which upgrades the disease to T4. ${ }^{9-11}$ Identification of non mass like enhancement along with the index lesion increases the total span of the disease in the breast as compared with the clinical examination and may be a predictor of the presence of extratumoral DCIS or extensive intraductal component. DCE MR also helps in the nodal staging of breast cancer by showing the levels of suspicious axillary nodes as well as in detection of internal mammary nodes and supraclavicular nodes. The metastatic involvement of the axillary nodes is suggested by certain features such as rounding of shape, eccentric cortical thickening, compression or partial or complete effacement of fatty hilum. This information is necessary for treatment decision in choosing between breast conservation and mastectomy, preoperative and post operative chemotherapy or hormonal therapy, sentinel lymph node biopsy and axillary lymph node dissection. ${ }^{1}$ However with all its capabilities and high sensitivity to identify and stage breast cancer, 
studies have shown that MRI has a low specificity in terms of high false positive rates and in identifying and specifically characterizing the extratumoral and non-mass forming DCIS. ${ }^{12}$

\section{Our study was aimed}

i. To determine the accuracy of MRI in evaluating the multicentric/ multifocal breast cancer and its significance as a roadmap in treatment planning.

ii. To study those interpretation criteria of MRI that serve to increase its specificity in patients with non mass like enhancement and therefore bring down false positive results.

iii. To study its role in predicting the nodal status in patients diagnosed with breast cancer.

\section{Materials and methods}

This is a retrospective study, conducted in the Department of Radio diagnosis; Max Super Speciality Hospital, New Delhi, from September 2011 to June 2014. The study was conducted after the approval of Institutional Review board. The Institutional Ethics committee waivered informed consent, this being a retrospective study. 156 female patients with breast cancer, who underwent MRI in our department for assessment of the disease prior to treatment planning and subsequently underwent treatment in the hospital's Cancer centre, were included in our study. These also included 14 post lumpectomy patients operated elsewhere who either had positive surgical margins or were detected with breast cancer on lumpectomy specimen and MRI was conducted for assessment of presence of residual disease and its extent for further treatment planning. There were 14 posts Neoadjuvant chemotherapy patients in whom MRI was done to take a decision for wide excision or mastectomy depending upon the response.

\section{Imaging}

Magnetic resonance imaging

Equipment: MR imaging of the breast was performed on 3.0 Tesla MRI units (Discovery 750 by GE Healthcare USA). All patients were imaged in the prone position using dedicated 8 channel bilateral breast coils and providing adequate compression.

Protocol for breast MRI: After adequate positioning of patient on a dedicated phased array breast coil on a $3 \mathrm{~T}$ scanner, Axial T2FSE IDEAL and Non fat suppressed Axial T1 sequences are obtained with slice thickness of $3 \mathrm{~mm}$ and $1 \mathrm{~mm}$ spacing with FOV of 36 . Diffusion weighted images were obtained at b value of 1000 and in some cases also at b 1500 in cases of glandular breast or those with fibrocystic breasts.

Following this a dynamic transverse 3D fat suppressed spoiled gradient echo volume acquisition was acquired through both the breasts prior to and six times sequentially following intravenous administration of $0.1 \mathrm{mmol} / \mathrm{kg}$ Gd-DTPA at $3 \mathrm{ml} / \mathrm{sec}$ bolus with no scan delay. This was followed by a delayed high resolution contrast enhanced sagittal sequence. These images were then subtracted and reconstructed in multiple planes. Kinetic analysis was done in region of interest. Colour maps were also viewed on the work station using specialized software.

Interpretation of MRI: Evaluation was done using ACR MRI BIRADS Lexicon
A. Mass lesion: Assessed for its size, morphology in terms of margins, shape signal characteristics, ADC value and enhancement characteristics (homogenous/heterogeneous/ring enhancement/ dark internal septations) and kinetic curves. Number of lesions (>2 lesions taken as multiple) was also assessed.

B. Non mass enhancement: Assessed for its distribution (focal/ linear/segmental/regional/diffuse) and internal enhancement characteristics (homogenous/heterogeneous/clustered ring clumped).

C. Lymph nodes: Assessed for eccentric cortical thickening/partial or complete effacement of fatty hilum and perinodal fat stranding.

These patients eventually underwent mastectomy or wide decision with sentinel lymph node biopsy or axillary lymph node dissection. The final specimen pathology report was correlated with MRI findings and variables studied were number of lesions reported, size of the largest single tumor reported, intratumoral DCIS present/absent, extratumoral DCIS/LCIS, extensive intraductal component and nodal metastasis.

\section{Results}

Our study included 156 patients, who were ranged between 25 and $70 y r s$. In our study, clinically and mammographically occult additional lesions were detected on MRI in $66 / 156$ patients (42.3\%). Of these in $34 / 66(51.5 \%)$ patients, there was non mass enhancement associated with the index lesion thereby increasing the total span of involvement on MRI. (Figure 1) (Figure 2) 25/34(73.5\%) of these lesions were confirmed on histopathology as extratumoral DCIS/ LCIS (Figure 3) or extensive intraductal component while in 9/34 patients the histopathology showed benign breast disease.

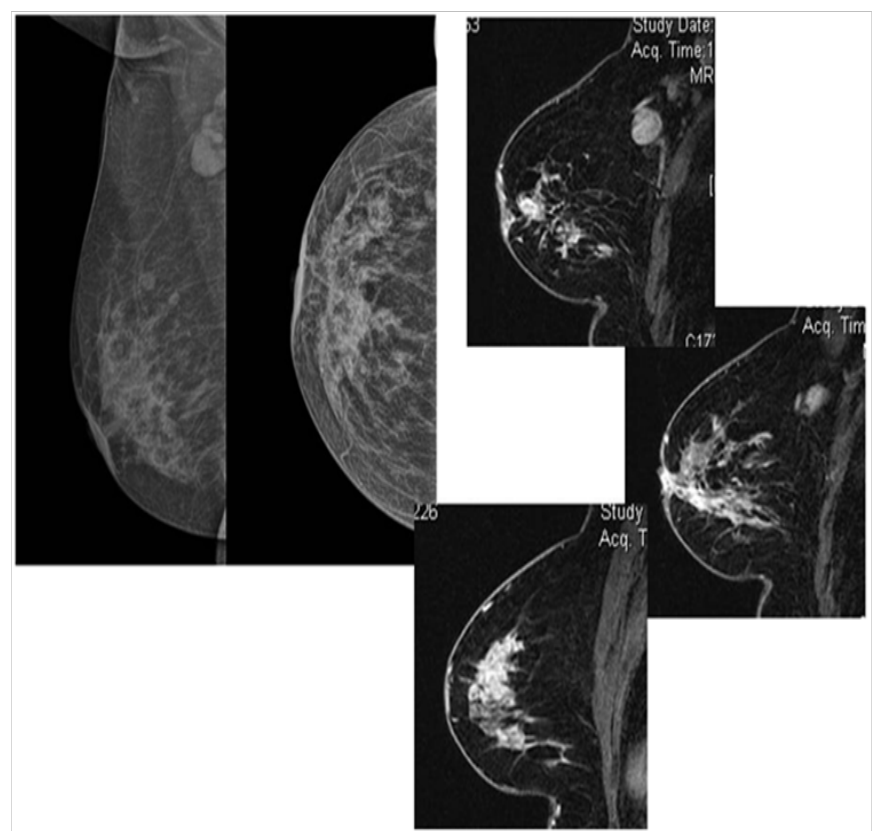

Figure I Patient came with palpable axillary nodes and mild nipple retraction. XRAY Mammogram (A,B...MLO and CC views)showed few micro calcify foci in lower inner quadrant of the right breast with axillary adenopathy. Mild nipple retraction is also noted DCE-MRI showed a large area of clumped NME extending from the upper outer quadrant to the lower inner quadrant and showing type 2 kinetics (D). The enhancement was also involving the nipple with its retraction $(C)$. 

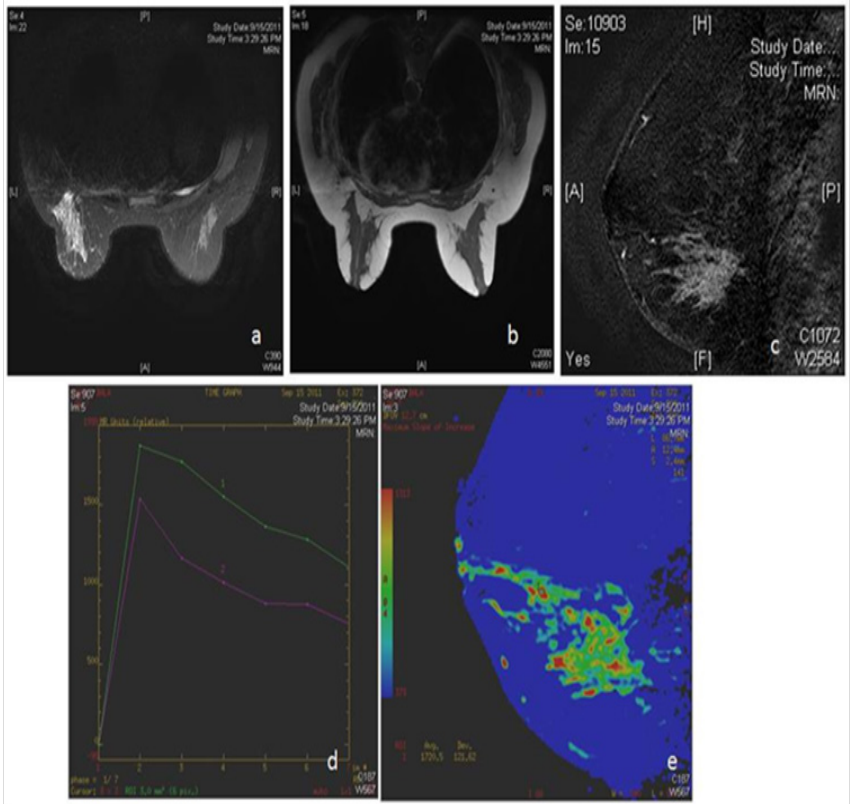

Figure $2 \mathrm{MRI}$ in patient with bloody nipple discharge shows a large area of non mass like enhancement in lower outer quadrant of the left breast (C,E) with type 3 kinetics. Histopathology revealed high grade DCIS.

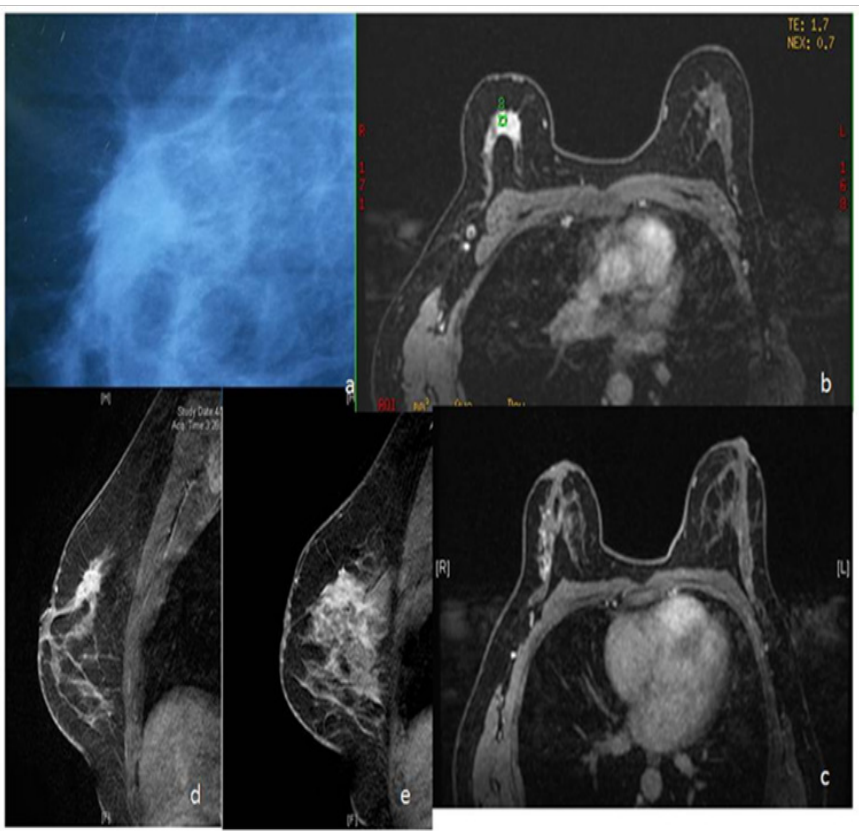

Figure 3 Lesion detected on screening mammography in right breast (A) On MRI, besides the primary lesion, there is linear nodular NMLE extending further laterally $(C, D, E)$. Mastectomy specimen revealed infiltrating ductal carcinoma with DCIS with invasive lobular carcinoma with LCIS.

Of the rest 32 patients with additional disease, 22 patients had multifocal/multicentric disease with non mass like enhancement and 10 patients had multifocal/multicentric disease without any associated non mass like enhancement (Figure 4). 2 of these patients had bilateral disease (Figure 5) and 1 was a diagnosed case of paget's disease of the nipple (Figure 6). In 27/32(84.3\%) patients, MRI results corroborated with the final histopathology results and in $5 / 32$ patients, there were false positive results. Our study showed that MRI had Sn: $86 \%$, Sp: $89 \%$, PPV: $60 \%$, NPV: $97 \%$ for multifocal/multicentric tumours when correlated with histopathology (Figure 7).
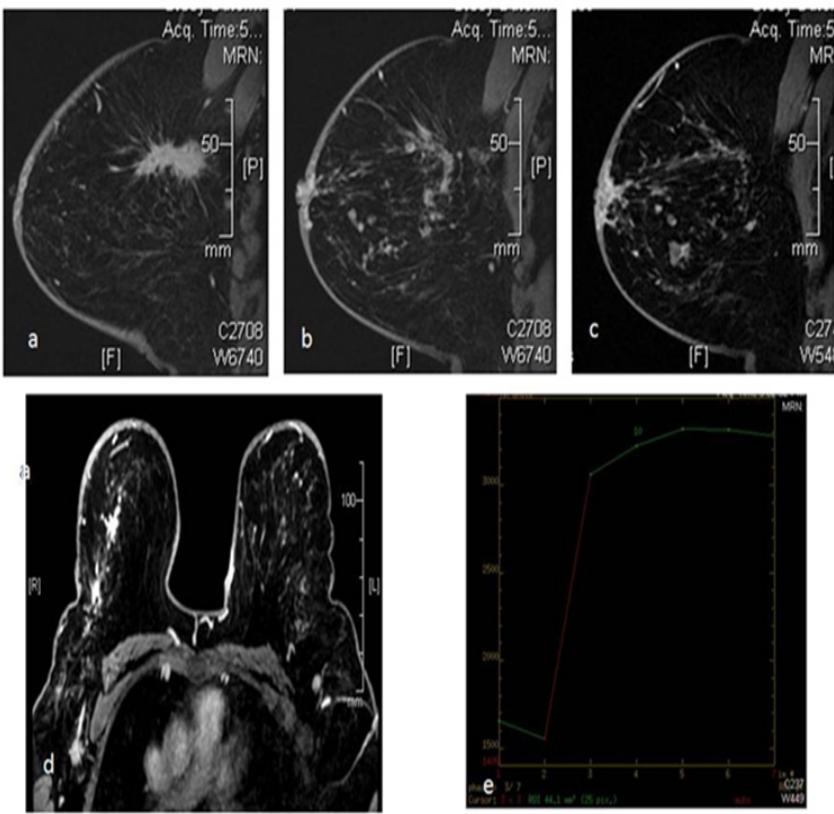

Figure $439 y$ r old female patient came with palpable lump in right breast, along with vague nodularity in the outer half of the breast. DCE MRI shows an oblong spiculated mass lesion in the upper outer quadrant of the right breast (A) with multiple linear and nodular areas of enhancement which is extending up to the nipple. There is involvement of the skin of nipple areola complex $(B, C, D)$. Nodular areas were seen extending to the lower outer quadrant of the breast as well. This lesion showed type 2 kinetics (E). The patient was treated with neoadjuvant chemotherapy followed by surgery.

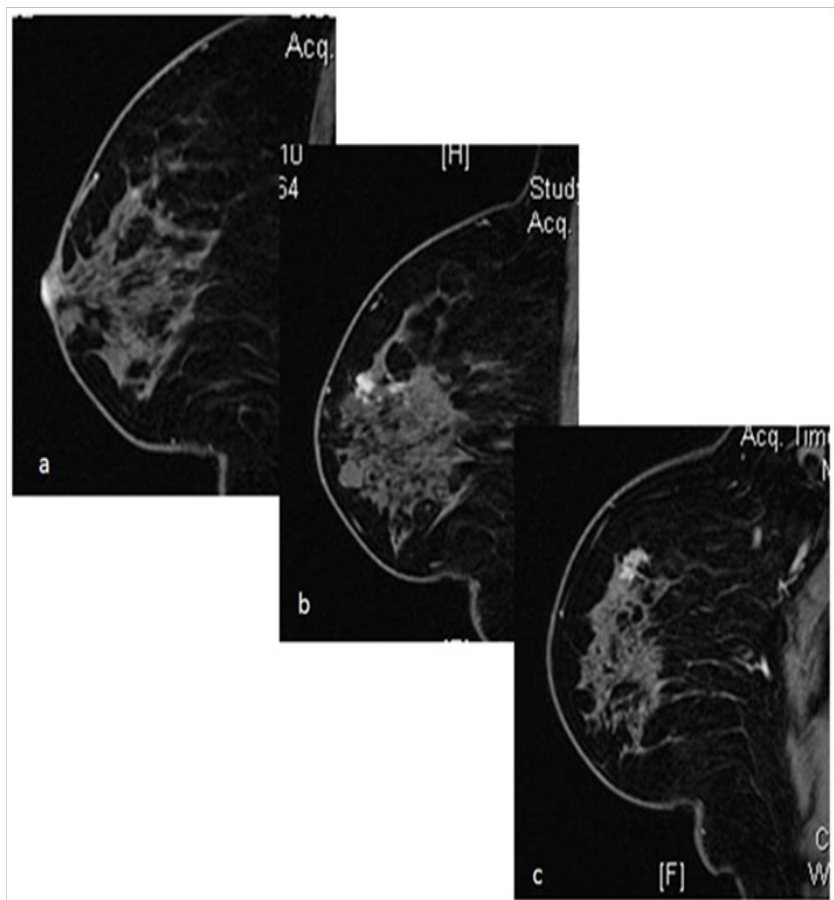

Figure 5 Case of Paget's disease of the nipple and proven on nipple biopsy. Preoperative MRI revealed abnormal enhancement in the nipple (A) with multiple nodular areas of enhancement with type 2 and type 3 kinetics in the underlying breast parenchyma, located at a distance more than $2 \mathrm{~cm}$ from the nipple areola complex $(\mathrm{B}, \mathrm{C})$. Ultrasound guided biopsy revealed IDC with DCIS in one of the larger nodules sampled. Patient was treated with mastectomy. This case highlights the role of MRI in treatment planning of Paget's disease. 

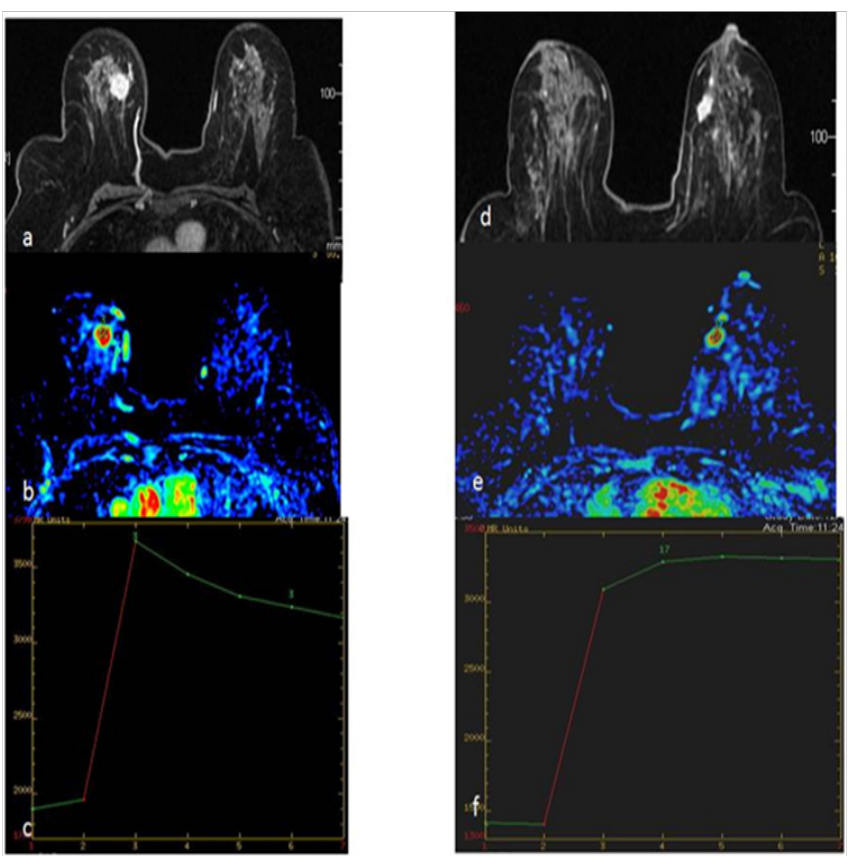

Figure $646 y r$ old woman presented with palpable lump in her right breast. DCE MRI done for preoperative staging shows intensely enhancing mass lesion in right breast with type 3 kinetics $(A, B, C)$ with similar smaller lesion also seen in left breast showing type 2 plateau kinetics (D,E,F). USG guided biopsy of both lesions revealed Infiltrating Ductal Carcinoma. The patient also had enlarged suspicious nodes in right axilla which were proven metastatic on final histopathology.
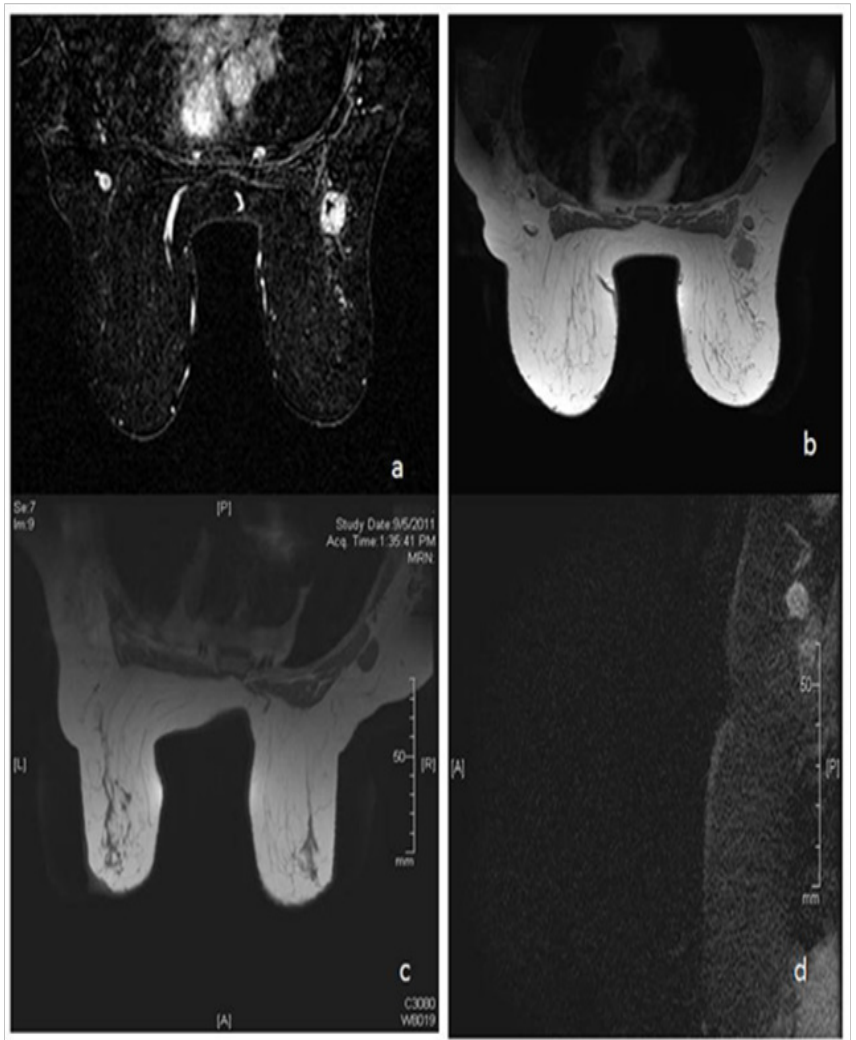

Figure 7 There is spiculated mass lesion in right breast $(A)$, with adjoining node showing cortical thickening with partial effacement of fatty hilum (B). C and $D$ show enlarged node with complete effacement of fatty hilum and mildly irregular margins.

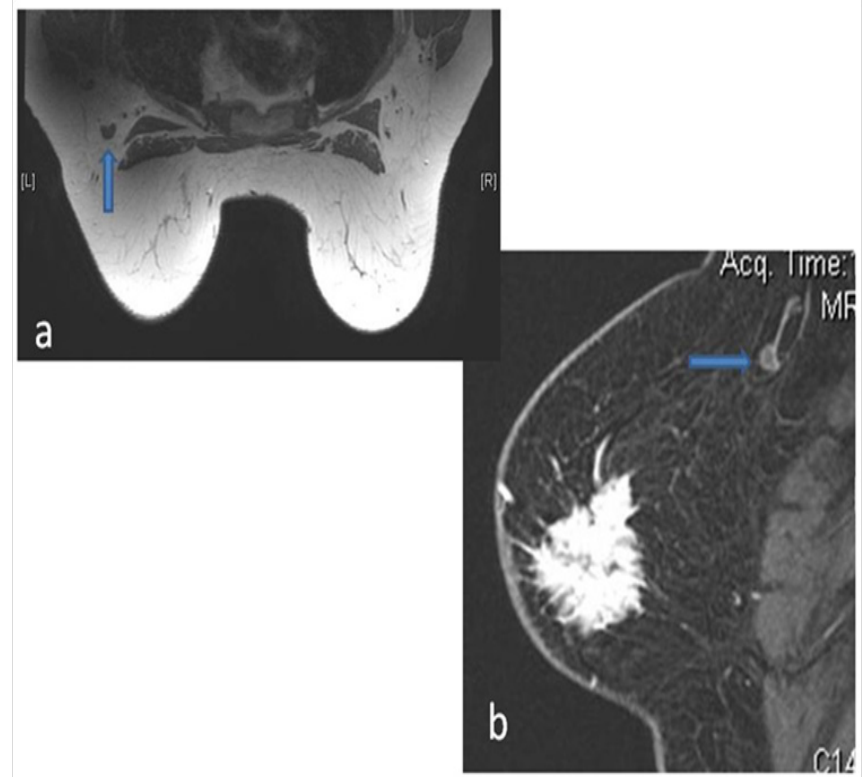

Figure 8 Eccentric cortical thickening without fatty hilum replacement as seen in precontrast TIW image (A) and post contrast sagittal VIBRANT image (B).

We assessed role of MRI in detection of metastatic axillary adenopathy by studying two criteria i.e. eccentric cortical thickening and effacement of fatty hilum. We found that in 58/156 patients, there was effacement of fatty hilum and 50/58 of these cases showed metastatic involvement on histopathology while in $8 / 58$ patients histopathology showed only reactive adenopathy. MRI for fatty hilum effacement showed Sn: 65\%, Sp: 89\%, PPV: 86\%, NPV: 73\%. When cortical thickening in the nodes was taken as the criteria of involvement, 91/156 patients had cortical thickening of which 67/91 were positive for metastasis on histopathology and 24/91 were negative. Cortical thickening without fatty hilum effacement showed Sn: 86\%, Sp: 69\%, PPV: 73\%, NPV: 84\%. The results have been summarised in Table 1.

Table I Results of our study in nutshell

\begin{tabular}{|c|c|c|c|c|}
\hline & Sensitivity & Specificity & PPV & NPV \\
\hline $\begin{array}{l}\text { NME with Extratumoral } \\
\text { DCIS on HSP }\end{array}$ & $79 \%$ & $86 \%$ & $76 \%$ & $88 \%$ \\
\hline NME with EIC & $74 \%$ & $76 \%$ & $52 \%$ & $89 \%$ \\
\hline $\begin{array}{l}\text { MF/MC with multiple } \\
\text { lesions on } \mathrm{HSP}\end{array}$ & $86 \%$ & $89 \%$ & $60 \%$ & $97 \%$ \\
\hline \multicolumn{5}{|l|}{ NME with both } \\
\hline $\begin{array}{l}\text { Extratumoral DCIS } \\
\text { and EIC }\end{array}$ & $89 \%$ & $78 \%$ & $50 \%$ & $97 \%$ \\
\hline $\begin{array}{l}\text { Lymph nodes with } \\
\text { effaced fatty hila and } \\
\text { HSP correlation }\end{array}$ & $65 \%$ & $89 \%$ & $86 \%$ & $73 \%$ \\
\hline $\begin{array}{l}\text { Lymphnodes with } \\
\text { cortical thickening and } \\
\text { HSP correlation }\end{array}$ & $86 \%$ & $69 \%$ & $73 \%$ & $84 \%$ \\
\hline
\end{tabular}




\section{Discussion}

In our study of 156 patients, who were ranged between 25 and $70 \mathrm{yrs}$. The size of the primary lesion as measured on MRI correlated well with the histopathological size with correlation coefficient of $\mathrm{r}=0.91$. We compared the maximum dimension measured on MRI with the maximum dimension on pathology including $0.5 \mathrm{~cm}$ variation, although some studies have suggested that comparing the average size or the tumor volume gives more accurate results especially in patients on neoadjuvant chemotherapy.

\section{Detection of additional lesions on MRI with or without non mass enhancement (NME)}

Our study showed that MRI could detect additional clinically and mammographically occult lesions in $66 / 156$ i.e. $42.3 \%$ patients which included multifocal/multicentric lesions as well as NME, showing sensitivity and specificity of $86 \%$ and $89 \%$ respectively.

In various studies done by Liberman et al., ${ }^{9}$ Mumtaz $\mathrm{H}$ et al., ${ }^{11}$ Lee JM et al., ${ }^{13}$ Boetes $\mathrm{C}$ et al., ${ }^{14}$ the sensitivity of MRI in detection of additional lesions was shown to be $33.3 \%, 11.8 \%, 27.1 \%, 13.3 \%$ and $16.1 \%$ respectively. These variations are mainly contributed by the patient population and type of malignancy as seen in the study by Rodenko et al., ${ }^{15}$ who saw that in their study all the 20 patients studied had Invasive lobular carcinoma and had multifocal/multicentric disease. In our study, 5/66 patients had ILC, 11/66 had predominant DCIS. Robert L Gutierrez et al., ${ }^{16}$ reviewed 592 newly diagnosed patients of breast cancer who underwent preoperative breast MRI (570 patients comprised the analysis set). They found that 152 of 570 patients $(27 \%)$ had one or more additional suspicious lesions on MRI besides the index lesion. 67/152 patients who underwent biopsy had additional cancer diagnosed. Nehmat Houssami et al., ${ }^{5}$ in a systematic review and meta analysis of 19 studies for the accuracy of MRI in detection of multifocal/multicentric cancer not identified on conventional imaging observed MRI detects additional disease in $16 \%$ cases. Sardanelli et al. ${ }^{17}$ compared MRI and Mammography in detection of multifocal, multicentric cancer foci and found that the overall sensitivity was $66 \%(124 / 188)$ for mammography and $81 \%(152 / 188)$ for MRI $(p<0.001) ; 72 \%(113 / 158)$ and $89 \%(140 / 158)$ for invasive foci $(p<0.001)$; and $37 \%(11 / 30)$ and $40 \%(12 / 30)$ for in situ foci. Mammography and MRI missed 64 and 36 malignant foci respectively. Our study did not have direct comparison between MRI and mammography in detection of additional lesions, but overall sensitivity of MRI was found to be $86 \%$.

One of the goals of our study was to optimally characterise nonmass enhancement as benign and malignant according to their distribution and internal enhancement patterns, mainly based on BIRADs descriptors in order to reduce false positive rates and also to assess tumor extent. In practice various studies show different criteria and characteristics of NME to characterize it as benign and malignant. These criteria are distribution and internal enhancement pattern mainly while some studies have also included kinetic curve (type $1,2,3$ ) and signal enhancement ratio (SER).$^{18}$ In our study we used distribution and internal enhancement pattern to grade the NME as malignant or benign.

In our study, 34/156 patients had NME of which $73.5 \%$ were confirmed on histopathology as DCIS/LCIS or EIC. In rest of the patients NME was benign on histopathology. Our analysis on the distribution and internal enhancement characteristics NME showed the most common pattern of NMLE associated with extratumoral DCIS and/EIC was clumped/clustered ring enhancement in segmental and regional distribution followed by linear distribution.

Homogeneous enhancement in linear pattern showed least number of malignant cases. Clumped/clustered ring enhancement in linear distribution was most common in cases of Infiltrating Lobular carcinoma Table 2. Hiramatsu et al. ${ }^{19}$ found that linear and/or spotty enhancement on MRI suggested the presence of DCIS or intraductal spread in the area surrounding the invasive cancer. In their study of 72 patients, 50 showed linear and/or spotty enhancement on MRI and 41 of those 50 patients had DCIS or intraductal spread. The sensitivity, specificity and accuracy for detecting intraductal spread on MRI were $89 \%, 82 \%$ and $81 \%$, respectively. The surgical decision of MRM purely on the basis of MRI findings was present in 48 patients. In study by Wai Ong et al., ${ }^{20}$ the most common distribution was ducal/linear $(48.4 \%)$ and most common internal enhancement pattern was clumped. While Wilhelm et al. ${ }^{21}$ found clumped pattern of enhancement and segmental distribution of NME had the highest sensitivities of $40.5 \%$ and $23.5 \%$ respectively for malignancy. Our study findings are well in correlation with these studies.

Table 2 NMLE pattern correlation with extratumoral DCIS and/or EIC on histopathology

\begin{tabular}{lll}
\hline & True positive & False positive \\
\hline Clumped segmental & $33 / 66(50 \%)$ & $3 / 66(4.5 \%)$ \\
Heterogenous linear & $3 / 66(4.5 \%)$ & $5 / 66(7.5 \%)$ \\
Clumped Linear & $5 / 66(7.5 \%)$ & $1 / 66(1.5 \%)$ \\
Homogenous linear & $1 / 66(1.5 \%)$ & $15 / 66(22 \%)$ \\
\hline
\end{tabular}

Ballesio et al., ${ }^{22}$ in their study on 94 patients, done in 2014, found that $52.4 \%$ showed regional pattern of distribution, $27.7 \%$ had segmental pattern and $20.2 \%$ a ducal distribution of NME. When the findings on MRI were correlated with final histopathology of the postoperative specimen, our study showed $\mathrm{Sn}: 79 \%$, Sp: 86\%, PPV: 76\%, NPV: $88 \%$ for NME contributed by extratumoral DCIS, while Sn: $74 \%$, Sp: 76 , PPV: $52 \%$, NPV: $89 \%$ for NME due to extensive intraductal component (EIC) on histopathology. In our study 26 patients were such who had NME and on histopathology they had both EIC and Extratumoral DCIS.

The current treatment guidelines recommend wide excision or mastectomy for multifocal/multicentric disease; therefore identification of additional lesions on MRI does impact clinical treatment decisions. ${ }^{23}$ Although how much this extends into clinical and long term survival benefit is still a matter of debate. Clinical trials exist for both invasive and in situ carcinoma in which no significant difference in survival was found in patients treated with $\mathrm{BCT}$ or mastectomy. ${ }^{24-26}$ Both radiation therapy and adjuvant systemic chemotherapy contribute to low rates of cancer recurrence in treated breast.

Shell et al. ${ }^{27}$ in their study confirmed with their results that breast MRI is useful in detection of mammographically occult lesions in ipsilateral and contralateral breasts and detection of any contralateral malignant tumor substantially affects patient's treatment and is important for patient care. Punglia et al. ${ }^{28}$ in their study showed that improved local disease control with either more extensive surgery or whole breast radiation improves 15 year survival rate in patients with 
extensive local disease. The available systemic therapies are mainly effective against microscopic disease.

Preoperative knowledge of tumor extent may also lead to decreased need for reexcision because of adequate surgical margins as presented by Comstock et al. ${ }^{29}$ In many cases even negative findings on MRI play a significant role. In our study MRI showed NPV of $97 \%$ in detection of Multifocal/multicentric disease. Identification of multicentric disease/characteristic NME pointing towards presence of extratumoral in situ disease and extensive residual disease help in identifying the patients who would benefit from mastectomy rather than breast conservation surgery. In our study analysis, 27 underwent mastectomy and 7 had wide local excision on the basis of MRI findings. We also compared these results with other studies as in Table $3.30-32$

Table 3 Studies conducted by various authors

\begin{tabular}{lll}
\hline Study & $\begin{array}{l}\text { MRI changed decision } \\
\text { to mastectomy }\end{array}$ & $\begin{array}{l}\text { Mastectomy done with } \\
\text { false positive findings }\end{array}$ \\
\hline Bagley et al. $^{30}$ & Mastectomy 6/27(22.2\%) & Mastectomy 3/27(1I.1\%) \\
Berg et al. ${ }^{31}$ & Mastectomy 6/96(6.3\%) & Mastectomy 5/96(5.2\%) \\
Godinez et al. ${ }^{32}$ & Mastectomy $8 / 79(10.1 \%)$ & Mastectomy 2/79(2.5\%) \\
Our study & $\begin{array}{l}\text { Mastectomy } \\
42 / 156(26.9 \%)\end{array}$ & Mastectomy $1 \mathrm{I} / 56(0.07 \%)$ \\
\hline
\end{tabular}

\section{Axillary lymphadenopathy}

MRI imaging done with dedicated breast coil and thin section imaging of the axilla may act as an effective tool in determining the axillary lymph node metastasis in newly diagnosed cases of breast cancer. Imaging of the axilla requires thin section imaging of the axilla with patient in prone comfortable position. In our centre we used dedicated breast coil at 3 Tesla system with $2 \mathrm{~mm}$ thickness slices of the axilla both precontrast T1W non fat sat and post contrast T1W non fat sat. MRI provides visualization and comparison of bilateral axilla without any appreciable increase in scan time. Unenhanced $\mathrm{T} 1 \mathrm{~W}$ images without fat suppression help in identification of axillary nodes with their abnormal characteristics in metastasis.

The major criteria we studied in classifying a node as suspicious for metastasis were eccentric cortical thickening, effacement of fatty hilum, change in the shape of the enlarged nodes and perinodal fat stranding. Our study showed that if only complete effacement of fatty hilum was included MRI had sensitivity of $69.4 \%$, specificity of $90.9 \%$, PPV of $86.27 \%$ and NPV of $78.65 \%$. Inclusion of eccentric cortical thickening with cut off of $3 \mathrm{~mm}$. increased the sensitivity of $93.94 \%$, specificity to $72.9 \%$ and NPV and PPV to $93.10 \%$ and $75.61 \%$ respectively (Figure 8 ).

Scaranelo et al..$^{33}$ in their study achieved sensitivity and specificity of $88 \%$ and $82 \%$ respectively when studying the $\mathrm{T} 1 \mathrm{~W}$ imaging characteristics of involved nodes. Their study however also included the ADC value of metastatic nodes and showed significant difference between the ADC of malignant and benign nodes. This was also seen in our study but ADC could not consider as a reliable criteria in clinical context since even reactive or inflamed nodes also at times showed low ADC value. Perinodal fat stranding when present was seen as a highly sensitive criteria of nodal involvement with extra capsular extension of disease.

Mortellaro et al. ${ }^{34}$ in their study concluded that presence of any axillary lymph node with no fatty hilum and the number of nodes with no fatty hilum on MR significantly correlated with pathologic node positivity $(\mathrm{P}=0.04)$; while kinetics, node number, and node size did not correlate. This is also well in correlation with our study. In study by Luciani et al., ${ }^{35}$ also eccentric or irregular cortical thickening and non visualization of hilum was seen as having a high positive predictive value for nodal metastasis. They also showed that irregular nodal margin was correlating well with extracapsular extension.

Murray et al. ${ }^{36}$ studied nodal enhancement index and nodal area as criteria of nodal involvement and showed that nodal enhancement index of $>21 \%$ and nodal area of $>0.4 \mathrm{~cm}$ had sensitivity of $100 \%$ in prediction of axillary nodal metastasis with low specificity of $56 \%$. The limitation of our study was that we could not obtain meaningful comparison of concordance of size between MRI and HPE based on different histologies as majority of the lesions were IDC. Also we need to include other diagnostic criteria besides effacement of fatty hilum and cortical thickening before we could confidently state the positive role of MRI in predicting axillary node metastasis in breast cancer patients.

\section{Conclusion}

Our study concluded that MRI has a high sensitivity in early detection of breast cancer including the detection of multicentric/ multifocal lesions in the same as well as contralateral breast which are occult on conventional imaging techniques. Identification of multiple lesions in different quadrants of the breast helps in treatment decisions in which either the patient is treated with mastectomy or with neoadjuvant chemotherapy. However multiple tumors in the same quadrant within $2 \mathrm{~cm}$ of the primary lesion can be still treated with wide excision with subsequent whole breast radiation as the protocol followed in our hospital's cancer centre.

It also has a high sensitivity in detection of non mass like enhancement. Our study showed that non mass enhancement in segmental or regional distribution with clumped internal enhancement has high specificity in detection of DCIS or extensive intraductal component. Identification of these patterns can help in differentiating benign and malignant NME and also in diagnosis of DCIS

Although statistically significant correlation was seen between effacement of fatty hilum and axillary nodal metastasis, it has low sensitivity and further studies with inclusion of other specific criteria are required before MRI can be used as a definitive modality for prediction of nodal metastasis in these patients.

Given the above mentioned potential of MRI in evaluation of breast cancer, MRI may be added to the preoperative imaging work-up of breast cancer patients as an aid in surgical and definitive treatment. Breast MRI has a definite potential to reduce the number of surgical procedures to obtain negative margins of resection or to convert patients from planned breast-conservation therapy (BCT) to mastectomy.

One of the major limitations of MRI of the breast in breast cancer staging is false-positive enhancement as seen in fibroadenomas, fat necrosis and fibrocystic changes resulting in being results on biopsy on the MRI detected suspicious lesions. This may result in increased cost, patient anxiety and increased rate of mastectomies. Balancing 
the known pitfalls of breast MRI and its false positives with the clinical goal of accurate assessment of extent of disease is a subject of continued investigation.

\section{Acknowledgements}

None.

\section{Conflict of interest}

Author declares that there is no conflict of interest.

\section{References}

1. Lee SC, Jain PA, Jethwa SC, et al. Radiologist's role in breast cancer staging: providing key information for clinicians. Radiographics. 2014;34(2):330-342.

2. Orel SG, Schnall MD, Powell CM, et al. Staging of suspected breast cancer: effect of MR imaging and MR-guided biopsy. Radiology. 1995;196(1):115-122.

3. Morris EA, Schwartz LH, Dershaw DD, et al. MR imaging of the breast in patients with occult primary breast carcinoma. Radiology. 1997;205(2):437-440.

4. Esserman L, Hylton N, Yassa L, et al. Utility of magnetic resonance imaging in the management of breast cancer: evidence for improved preoperative staging. J Clin Oncol. 1999;17(1):110-119.

5. Houssami N, Ciatto S, Macaskill P, et al. Accuracy and surgical impact of magnetic resonance imaging in breast cancer staging: systematic review and meta-analysis in detection of multifocal and multicentric cancer. $J$ Clin Oncol. 2008;26(19):3248-3258.

6. Weinstein SP, Orel SG, Heller R, et al. MR imaging of the breast in patients with invasive lobular carcinoma. AJR Am J Roentgenol. 2001;176(2):399-406

7. Fischer U, Baum F, Nagel LS. Preoperative MR imaging in patients with breast cancer: preoperative staging, effects on recurrence rates, and outcome analysis. Magn Reson Imaging Clin N Am. 2006;14(3):351-362.

8. Salem DS, Kamal RM, Mansour SM, et al. Breast imaging in the young: the role of magnetic resonance imaging in breast cancer screening, diagnosis and follow-up. J Thorac Dis. 2013;5(1):S9-S18.

9. Liberman L. Breast MR imaging in assessing extent of disease. Magn Reson Imaging Clin N Am. 2006;14(3):339-349.

10. Tillman GF, Orel SG, Schnall MD, et al. Effect of breast magnetic resonance imaging on the clinical management of women with early stage breast carcinoma. J Clin Oncol. 2002;20(16):3413-3423.

11. Mumtaz H, Hall-Craggs MA, Davidson T, et al. Staging of symptomatic primary breast cancer with MR imaging. AJR Am J Roentgenol. 1997; 169(2):417-424.

12. Klostergaard J, Parga K, Raptis RG. Current and Future Applications of Magnetic Resonance Imaging (MRI) to Breast and Ovarian Cancer Patient Management. PR Health Sci J. 2010;29(3):223-231.

13. Lee JM, Orel SG, Czerniecki BJ, et al. MRI before reexcision surgery in patients with breast cancer. AJR Am J Roentgenol. 2004;182(2):473-480.

14. Boetes C, Mus RD, Holland R, et al. Breast tumors: comparative accuracy of MR imaging relative to mammography and US for demonstrating extent. Radiology. 1995;197(3):743-747.

15. Rodenko GN, Harms SE, Pruneda JM, et al. MR imaging in the management before surgery of lobular carcinoma of the breast: correlation with pathology. AJR Am J Roentgenol. 1996;167(6):1415-1419.
16. Gutierrez RL, DeMartini WB, Silbergeld JJ, et al. High cancer yield and positive predictive value: outcomes at a centre routinely using preoperatibe breast MRI for staging. AJR Am J Roentgenol. 2011;196 (1):W93W99.

17. Sardanelli F, Iozzelli A, Fausto A. MR imaging of the breast: indications, established technique and new directions. Eur Radiol, 2003;13(3):N28N36.

18. Newstead GM. MR Imaging of Ductal Carcinoma In Situ. Magn Reson Imaging Clin N Am. 2010;18(2):225-240.

19. Hiramatsu H, Enomoto K, Ikeda T, et al. The role of contrast-enhanced high resolution MRI in the surgical planning of breast cancer. Breast Cancer. 1997;4(4):285-290.

20. Wai Ong EM, Raza S, Chikarmane SA, et al. Non mass like enhancement in breast MRI: Correlating BIRADS- based Characterization with pathology. Radiological Society of North America 2009 Scientific Assembly and Annual Meeting. Chicago; 2009.

21. Wlihelm A, McDonough MD, DePeri ER. Malignancy rates of nonmass like nehancement on breast magnetic resonance imaging using aAmerican College of Radiology Breast Imaging Reposting and Data System descriptors. Breast J. 2012;18(6):523-526.

22. Ballesio L, Di Pastena F, Gigli S, et al. Non mass like enhancement categories detected by breast MRI and histological findings. Eur Rev Med Pharmacol Sci. 2014;18(6):910-917.

23. Alessandro Neri, Daniele Marrelli, Tiziana Megha, et al. Clinical significance of multifocal and multicentric breast cancers and choice of surgical treatment: a retrospective study on a series of 1158 cases. BMC Surgery. 2015;15:1.

24. Onitilo AA, Engel JM, Stankowski RV, et al. Survival Comparisons for Breast Conserving Surgery and Mastectomy Revisited: Community Experience and the Role of Radiation Therapy. Clin Med Res. 2015;13(2):65-73.

25. Fischer U, Zachariae O, Baum F, et al. The influence of preoperative MRI of the breasts on recurrence rate in patients with breast cancer. Eur Radiol. 2004;14(10):1725-1731.

26. Goreclad JW, McCabe EB, Higgins JH, et al. Screening for recurrences in patients treated with breast conserving surgery: is there a role for MRI? Ann Surg Oncol. 2008;15(6):1703-1709.

27. Schell AM, Rosenkranz K, Lewis PJ. Role of breast MRI in the preoperative evaluation of patients with newly diagnosed breast cancer. AJR. 2009;192(5):1438-1444.

28. Punglia RS, Morrow M, Winer EP, et al. Local therapy and survival in breast cancer. $N$ Engl J Med. 2007;356(23):2399-2405.

29. Comstock C, Hunt P, Middleton M. Effect of preoperative MRI on mastectomy rates, lumpectomy negative margin rates and time to surgery in patients with known breast cancer. RSNA. 2007.

30. Frederick HB. The Role of Magnetic Resonance Imaging Mammography in the Surgical Management of the Index Breast Cancer. Arch Surg. 2004;139(4):380-383.

31. Berg WA, Gutierrez L, NessAiver MS, et al. Diagnostic Accuracy of Mammography, Clinical Examination, US, and MR Imaging in Preoperative Assessment of Breast Cancer. Radiology. 2004;233(3):830-849.

32. Godinez J, Gombos EC, Chikarmane SA, et al. Breast MRI in the evaluation of eligibility for accelerated partial breast irradiation. AJR Am J Roentgenol. 2008;191(1):272-277.

33. Anabel MS, Riham E, Lindsay MJ, et al. Accuracy of Unenhanced MRImaging in the Detection of Axillary Lymph Node Metastasis .Study of Reproducibility and Reliability. Radiology. 2012:262(2). 
34. Mortellaro VE, Marshall J, Singer L, et al. Magnetic resonance imaging for axillary staging in patients with breast cancer. J Magn Reson Imaging. 2009;30(2):309-312.

35. Luciani A, Dao TH, Lapeyre M, et al. Simultaneous bilateral breast and high-resolution axillary MRI of patients with breast cancer: preliminary results. AJR Am J Roentgenol. 2004;182(4):1059-1067.
36. Murray AD, Staff RT, Redpath TW, et al. Dynamic contrast enhanced MRI of the axilla in women with breast cancer: comparison with pathology of excised nodes. Br J Radiol. 2002;75(891):220-228. 